The Egyptian International Journal of Engineering Sciences \& Technology, Vol 8, No 1 (2004)

\title{
Flow resistance and flow forces through vegetated open channels
} atef abdelhameed elsaied

\begin{abstract}
The vegetation density in channel bed is the major factor that affects the resistance to flow. The flow resistance coefficients due to the flexible vegetation in open channels are studied in this paper in addition to the effect of flow characteristics on drag coefficient and drag force. The artificial vegetation (cylindrical plastic tubes) is studied under free and submerged conditions in a total of 150 runs for different vegetation intensities and different bed slopes. The dimensional analysis, supported by experimental results, is used to obtain a relationship between roughness coefficients and flow conditions. By using statistical analysis, empirical equations are obtained to calculate the roughness coefficient, the drag coefficient, and the drag force as a function of flow parameters
\end{abstract}

